\title{
Comparison of diagnostic values between CA125 combined with CA199 and ultrasound combined with CT in ovarian cancer
}

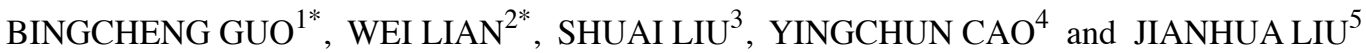 \\ ${ }^{1}$ Department of Ultrasound, Jining No. 1 People's Hospital, Jining, Shandong 272011; ${ }^{2}$ CT Room, \\ Yantaishan Hospital of Yantai, Yantai, Shandong 264001; Departments of ${ }^{3}$ Urology Surgery and ${ }^{4}$ Neurology, \\ People's Hospital of Zhangqiu District, Jinan, Shandong 250200; ${ }^{5}$ Clinical Laboratory, \\ The $5^{\text {th }}$ People's Hospital of Jinan, Jinan, Shandong 250000, P.R. China
}

Received August 27, 2018; Accepted April 9, 2019

DOI: $10.3892 / \mathrm{ol} .2019 .10264$

\begin{abstract}
Application values of CA125 combined with CA199 and ultrasound combined with computed tomography (CT) in the clinical diagnosis of ovarian cancer were compared. A retrospective analysis was performed on 168 ovarian cancer patients admitted to the Department of Gynecology in Jining No.1 People's Hospital from July 2013 to March 2016. Of the patients 107 with malignant tumors were in the malignant group, and 61 patients with benign tumors were in the benign group. Another 98 healthy controls in the same period were in the normal group. Chemiluminescence was used for the detection of levels of tumor markers CA125 and CA199 in the serum of all patients. CA125 combined with CA199 and color Doppler ultrasound combined with CT scan were used to diagnose and analyze the sensitivity, specificity, accuracy, and positive detection rate of ovarian cancer patients at different stages. The sensitivity, specificity and diagnostic coincidence rate of ultrasound combined with $\mathrm{CT}$ in the diagnosis of ovarian cancer were $97.20,80.32$ and $91.07 \%$, respectively, which were significantly higher than the 92.52, 73.77 and $85.71 \%$ of serum CA125 combined with CA199. The positive detection rate of ultrasound combined with CT in the early diagnosis of ovarian cancer was $93.55 \%$, higher than $83.87 \%$ of CA125 combined with CA199. The sensitivity, specificity, coincidence rate and positive detection rate of ultrasound combined with CT in the diagnosis were higher than those of CA125 combined with CA199. In the actual diagnosis process, these two diagnostic schemes can be selectively and
\end{abstract}

Correspondence to: Dr Jianhua Liu, Clinical Laboratory, The $5^{\text {th }}$ People's Hospital of Jinan, 24297 Jingshi Road, Jinan, Shandong 250000, P.R. China

E-mail: jqe2yv@163.com

${ }^{*}$ Contributed equally

Key words: CA125 combined with CA199, ultrasound combined with CT, ovarian cancer, diagnostic value comprehensively applied, so as to make a correct diagnosis, which is of great significance for reducing patient mortality.

\section{Introduction}

Ovarian cancer is a malignant tumor of the female reproductive system with the highest mortality. The incidence of ovarian cancer in recent years is getting higher due to the increasing pressure on life, which poses a serious threat to the health and lives of the majority of women (1). Patients have no obvious discomfort in the early stage of ovarian cancer, making it hard to detect, and the occurrence of obvious symptoms and the rapid progression of disease mean that they are in the advanced stage of ovarian cancer (2). However, there is still no effective method for the early diagnosis of ovarian cancer at diagnosis. Therefore, it is of great significance for the majority of women to receive an accurate early diagnosis of ovarian cancer, and then a timely and effective treatment.

At present, serum CA125 is an early and commonly used marker for the diagnosis of ovarian cancer in clinical practice. Studies have shown that CA125 level is increased in the serum of most ovarian cancer patients, but it has a low sensitivity in the early ovarian cancer patients. The CA125 level is increased not only in ovarian cancer, but also in some gynecological benign tumors and some other gynecological diseases $(3,4)$. Serum CA199 was first used in the detection of hepatobiliary pancreatic cancer and other digestive tract tumors due to its high sensitivity in the diagnosis of digestive tract tumors (5). However, in recent years, related research has found that CA199 is also increased in the serum of ovarian cancer patients (6). As people's awareness of tumor markers has increased, the combined detection of tumor markers has become an important issue. Ultrasound is the most commonly used method in gynecological examination, and plays a very important screening role in diagnosis because of non-invasiveness, high efficiency and high repeatability. Computed tomography (CT), especially CT-enhanced scanning, has been widely used in the diagnosis of ovarian cancer in recent years, and certain achievements have been obtained $(7,8)$.

In this study, the comparison of diagnostic values between CA125 combined with CA199 and ultrasound combined with 
CT was performed to find a better method for the early diagnosis of ovarian cancer to provide a better clinical reference.

\section{Patients and methods}

Basic information. A retrospective analysis was performed on 168 ovarian cancer patients admitted to the Department of Gynecology in Jining No. 1 People's Hospital (Jining, China) from July 2013 to March 2016. Among them, 107 patients with malignant tumors were in the malignant group, including 51 patients with serous cystadenocarcinoma, 7 patients with mucinous cystadenocarcinoma, 11 patients with endodermal sinus carcinoma, 4 patients with endometrioid carcinoma, 5 patients with transitional cell carcinoma, and 29 patients with borderline carcinoma. According to the staging, there were 31 patients in stage I-II and 76 patients in stage III-IV. Sixty-one patients with benign tumors were in the benign group. Furthermore, 98 healthy controls in the same period were in the normal group, aged from 36 to 52 years with a BMI less than $25 \mathrm{~kg} / \mathrm{m}^{2}$. There were no significant differences in the age and BMI of subjects among the three groups $(\mathrm{P}>0.05)$ (Table I).

Inclusion and exclusion criteria. Patients diagnosed with malignant ovarian tumors by pathological diagnosis were included in the malignant group. Patients diagnosed with benign ovarian tumors by pathological diagnosis were included in the benign group. Healthy controls who received physical examination were included in the normal group, aged from 36 to 52 years with a BMI less than $25 \mathrm{~kg} / \mathrm{m}^{2}$. Patients who had undergone radiotherapy and chemotherapy, patients with cognitive disorder and communication disorder, patients with severe liver and kidney function loss, and patients who did not cooperate with the examination were excluded. Patients with any other organic diseases in the normal group were excluded.

The study was approved by the Ethics Committee of Jining No. 1 People's Hospital. Patients who participated in this research had complete clinical data. All the patients and their families signed an informed consent form and cooperated with the medical staff to complete the relevant medical treatment.

Experimental instruments and kits. Siemens Acuson Sequoia 512 color Doppler ultrasound diagnostic instrument (probe frequency 2.5-6 MHz) from GE Healthcare (Chicago, IL, USA) was used for ultrasound diagnosis; Siemens 16-layer spiral CT machine was used for CT diagnosis with a scanning layer thickness of $5 \mathrm{~mm}$ (Siemens AG, Munich, Germany); the enhanced contrast agent was Ultravist 370 from Bayer Pharmaceuticals (Berlin, Germany). CA125 and CA99 kits were from Siemens AG. The detection principle of electrochemiluminescence (9) was used to detect CA125 and CA199 with a Beijing Yuande chemiluminescence immunoassay analyzer (output decibel was $<57.7 \mathrm{~dB}$ ) (Beijing Yuande Bio-Medical Engineering Co., Ltd., Beijing, China).

Methods. Approximately $5 \mathrm{ml}$ of fasting elbow venous blood was extracted from all subjects in the early morning, and centrifuged at $3,000 \mathrm{x}$ g for $10 \mathrm{~min}$ at $22^{\circ} \mathrm{C}$ to separate serum. CA125 and CA199 were detected in strict accordance with the kit instructions. The positive criteria for the two markers were CA125 >35 U/ml and CA199>37 U/ml, respectively. Then, gynecological ultrasound examination was performed on all patients. Patients were placed in a supine position, and the probe was covered with a sterile condom. The lump morphology, lump size, ascites and blood spectrum of patients were observed and recorded through the vagina. Criteria for the ultrasound diagnosis of ovarian cancer (10): Morphology and size of the ovary were elliptical, kidney or other irregular forms, and the echo and the thickness of the cyst wall were not uniform, with ascites in the abdominopelvic cavity. CT examination was performed after ultrasound examination. The enhanced scanning was performed after CT conventional plain scanning, and $90 \mathrm{ml}$ of the enhanced contrast agent was intravenously injected at a rate of $2.8 \mathrm{ml} / \mathrm{sec}$. Criteria for the CT diagnosis of ovarian cancer (11): Tumors were mainly cystic, often involving both sides, and the solid part was strengthened after the contrast enhancement. Low-density irregular necrosis was found in the solid lump, and the contrast was not enhanced. Metastatic nodules were found in the organs and pelvic wall of the abdominopelvic cavity. Ascites was formed, and lymph nodes in the abdominopelvic cavity were greater than $1 \mathrm{~cm}$.

Statistical analysis. SPSS 15.0 (SPSS, Inc., Chicago, IL, USA) statistical software was used for data processing. $\chi^{2}$ test was used for the comparison of counting data, and rank sum test for comparison between the two groups. Measurement data were expressed as mean \pm standard deviation, and one-way analysis of variance was used for comparison among multiple groups. The post hoc test used after ANOVA was Bonferroni. $\mathrm{P}<0.05$, indicates the difference is statistically significant.

\section{Results}

Detection results of CA125 and CA199 in three groups. There were differences in levels of CA125 and CA199 among the three groups $(\mathrm{P}<0.05)$. Patients in malignant and benign group had significantly higher serum CA125 and CA199 levels than those in normal group, with statistically significant differences $(\mathrm{P}<0.05)$. Patients in malignant group had significantly higher serum CA125 and CA199 levels than those in benign group $(\mathrm{P}<0.05)$ (Table II).

Analysis of diagnostic values of CA125, CA199, ultrasound and CT in 168 patients with ovarian tumor. Sensitivity, specificity, diagnostic coincidence rate, positive predictive value and negative predictive value of serum CA125 and CA199 alone in diagnosis of ovarian cancer were lower than those of ultrasound and CT alone. Misdiagnosis rate and missed diagnosis rate of CA125 and CA199 alone in the diagnosis of ovarian cancer were higher than those of ultrasound and CT alone (Table III).

Analysis of diagnostic values of CA125 combined with CA199 and ultrasound combined with CT in ovarian cancer. Sensitivity, specificity, diagnostic coincidence rate, positive coincidence rate and negative coincidence rate of CA125 combined with CA199 were 92.52, 73.77, 85.71, 86.09 and $71.43 \%$, respectively, lower than $97.20,80.32,91.07,89.66$ and $80.33 \%$ of ultrasound combined with CT. Misdiagnosis 
Table I. Basic data of the three groups of patients [n (\%)].

\begin{tabular}{|c|c|c|c|c|c|}
\hline Factors & Malignant group $(n=107)$ & Benign group $(n=61)$ & Normal group $(\mathrm{n}=98)$ & $t / \chi^{2}$ & P-value \\
\hline Age (years) & & & & 4.630 & 0.099 \\
\hline$\leq 45$ & $64(59.81)$ & $36(59.02)$ & 45 (45.92) & & \\
\hline$>45$ & $43(40.19)$ & $25(40.98)$ & $53(54.08)$ & & \\
\hline BMI $\left(\mathrm{kg} / \mathrm{m}^{2}\right)$ & & & & 0.225 & 0.894 \\
\hline$\leq 21$ & $59(55.14)$ & $32(52.46)$ & $51(52.04)$ & & \\
\hline$>21$ & $48(44.86)$ & $29(47.54)$ & $47(47.96)$ & & \\
\hline Marital status & & & & 0.915 & 0.633 \\
\hline Married & 89 (83.18) & $52(85.25)$ & 78 (79.59) & & \\
\hline Unmarried & $18(16.82)$ & $9(14.75)$ & $20(20.41)$ & & \\
\hline Birth history & & & & 0.368 & 0.832 \\
\hline Already fertile & $81(75.70)$ & $48(78.69)$ & $73(74.49)$ & & \\
\hline Not fertile & $26(24.30)$ & $13(21.31)$ & $25(25.51)$ & & \\
\hline Length of course (months) & $1.07 \pm 0.27$ & $1.13 \pm 0.29$ & - & 1.289 & 0.199 \\
\hline
\end{tabular}

Table II. Detection results of CA125 and CA199 in three groups.

\begin{tabular}{lcccc}
\hline Factors & $\begin{array}{c}\text { Malignant group } \\
(\mathrm{n}=107)\end{array}$ & $\begin{array}{c}\text { Benign group } \\
(\mathrm{n}=61)\end{array}$ & $\begin{array}{c}\text { Normal group } \\
(\mathrm{n}=68)\end{array}$ & $\mathrm{F}$ \\
\hline CA125 $(\mathrm{U}=\mathrm{ml})$ & $219.5 \pm 31.2$ & $35.8 \pm 8.2^{\mathrm{a}}$ & $11.5 \pm 5.6^{\mathrm{a}, \mathrm{b}}$ & 3.037 \\
$\mathrm{CA199}(\mathrm{U} / \mathrm{ml})$ & $81.3 \pm 23.1$ & $18.7 \pm 9.4^{\mathrm{a}}$ & $12.3 \pm 6.1^{\mathrm{a}, \mathrm{b}}$ & 571.9 \\
\hline
\end{tabular}

${ }^{\mathrm{a} C}$ Compared with malignant group, $\mathrm{P}<0.05$; ${ }^{\mathrm{b}}$ compared with benign group, $\mathrm{P}<0.05$.

Table III. Analysis of diagnostic values of CA125, CA199, ultrasound and CT in 168 cases of ovarian tumor patients [n (\%)].

\begin{tabular}{|c|c|c|c|c|}
\hline Factors & CA125 & CA199 & Ultrasound & $\mathrm{CT}$ \\
\hline Sensitivity & $83(77.57)$ & $73(68.22)$ & $85(79.44)$ & $89(83.18)$ \\
\hline Specificity & $49(80.33)$ & $43(70.49)$ & $50(81.97)$ & $52(85.25)$ \\
\hline Diagnostic coincidence rate & $132(78.57)$ & $116(69.05)$ & $135(80.36)$ & $141(83.93)$ \\
\hline Positive predictive value & $83(87.37)$ & $73(80.22)$ & $85(88.54)$ & $89(90.82)$ \\
\hline Negative predictive value & $49(67.12)$ & $43(55.84)$ & $50(69.44)$ & $52(74.29)$ \\
\hline Misdiagnosis rate & $12(19.67)$ & $18(29.51)$ & $11(18.03)$ & $9(14.75)$ \\
\hline Missed diagnosis rate & $24(22.43)$ & $34(31.78)$ & $22(20.56)$ & $18(16.82)$ \\
\hline
\end{tabular}

rate and missed diagnosis rate of CA125 combined with CA199 were 26.23 and $7.48 \%$, respectively, higher than the 19.67 and $2.80 \%$ of ultrasound combined with CT. It indicates that diagnostic value of ultrasound combined with CT is higher than that of CA125 combined with CA199 in ovarian cancer (Table IV).

Comparison of diagnostic positive rates of CA125, CA199, ultrasound and CT in ovarian cancer at different stages. Among patients with ovarian cancer in stage I-II, the positive rates of CA125 detection, CA199 detection, ultrasound detection, CT detection, CA125 combined with CA199, and ultrasound combined with CT were 38.71, 32.26, 61.29, 67.74, 83.87 and $93.55 \%$, respectively. Among patients with ovarian cancer in stage III-IV, the positive rates of CA125 detection, CA199 detection, ultrasound detection, CT detection, CA125 combined with CA199, and ultrasound combined with CT were 93.42, 82.89, 86.84, 89.47\%, 96.05 and $98.68 \%$, respectively. Positive rates of the combined detection were significantly higher than those of detection alone. Positive rate of ultrasound combined with $\mathrm{CT}$ was higher than that of CA125 combined with CA199, and that in detection of ovarian cancer in stage III-IV was higher than that in stage I-II (Table V). 
Table IV. Analysis of diagnostic values of CA125 combined with CA199 and ultrasound combined with CT in ovarian cancer $[\mathrm{n}(\%)]$.

\begin{tabular}{|c|c|c|c|c|c|c|c|}
\hline Factors & Sensitivity & Specificity & $\begin{array}{l}\text { Diagnostic } \\
\text { coincidence } \\
\text { rate }\end{array}$ & $\begin{array}{l}\text { Positive } \\
\text { predictive } \\
\text { value }\end{array}$ & $\begin{array}{l}\text { Negative } \\
\text { predictive } \\
\text { value }\end{array}$ & $\begin{array}{l}\text { Misdiagnosis } \\
\text { rate }\end{array}$ & $\begin{array}{l}\text { Missed } \\
\text { diagnosis } \\
\text { rate }\end{array}$ \\
\hline CA125 combined with CA199 & $99(92.52)$ & $45(73.77)$ & $144(85.71)$ & $99(86.09)$ & $45(71.43)$ & $16(26.23)$ & $8(7.48)$ \\
\hline Ultrasound combined with CT & $104(97.20)$ & $49(80.32)$ & $153(91.07)$ & $104(89.66)$ & $49(80.33)$ & $12(19.67)$ & $3(2.80)$ \\
\hline
\end{tabular}

Table V.Comparison of diagnostic positive rates of CA125, CA199, ultrasound and CT in ovarian cancer at different stages [n $(\%)$ ].

\begin{tabular}{lcccccc}
\hline Staging & CA125 & CA199 & Ultrasound & CT & $\begin{array}{c}\text { CA125 combined } \\
\text { with CA199 }\end{array}$ & $\begin{array}{c}\text { Ultrasound combined } \\
\text { with CT }\end{array}$ \\
\hline Stage I-II $(\mathrm{n}=31)$ & $12(38.71)$ & $10(32.26)$ & $19(61.29)$ & $21(67.74)$ & $26(83.87)$ & $29(93.55)$ \\
Stage III-IV $(\mathrm{n}=76))$ & $71(93.42)$ & $63(82.89)$ & $66(86.84)$ & $68(89.47)$ & $73(96.05)$ & $75(98.68)$ \\
\hline
\end{tabular}
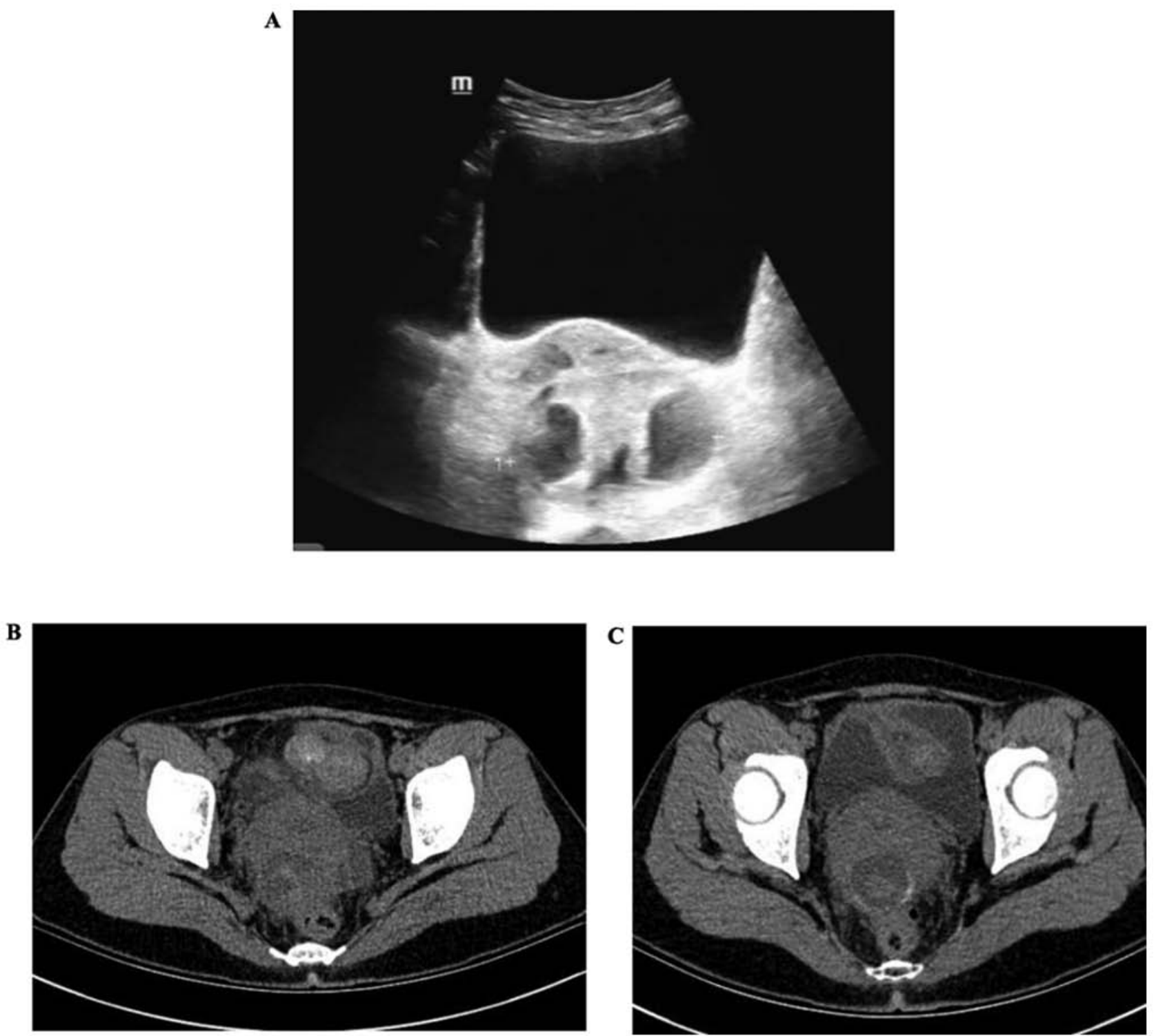

Figure 1. (A) Ultrasound image. Multiple solid capsules in the pelvic cavity with clear boundary and irregular shape. (B and C) CT images. Cystic solid lesions in the pelvic cavity with uneven density. Multiple patchy and spotty high-density shadows in the mass. 
Imaging features. Fig. 1A is an ultrasound image. There were multiple solid capsules in the pelvic cavity near the double attachment area. The larger one was approximately $7.9 \times 4.9 \mathrm{~cm}$, with clear boundary and irregular shape. CDFI: blood flow signals were found both inside and around. Fig. 1B and $\mathrm{C}$ are CT images. Large masses of cystic solid lesions were seen in the pelvic cavity surrounding the uterus. The density was uneven, and adjacent tissues were compressed. The boundary between the lesion and the intestinal canal was unclear. Multiple patchy and spotty high-density shadows were seen in the mass. The structure of the pelvic cavity was disordered, and there was no obvious increase in lymph node shadow in the pelvic cavity. The bladder was filled, and a small amount of fluid and density shadow was found in the pelvic cavity.

\section{Discussion}

Ovarian cancer is one of the malignant tumors that seriously threaten the health of women. It has no obvious features in the early stage and is easily overlooked, so most patients with ovarian cancer are in the advanced stage once diagnosed, which causes high mortality and less than $30 \%$ postoperative 5-year survival rate. If ovarian cancer could be detected early and then treated systematically, the 5-year survival rate will be no less than $90 \%$ (12). However, ovarian cancer is currently difficult to be diagnosed in the early stage due to its non-specific clinical performance, so the accurate diagnosis of early ovarian cancer patients is very important in clinical practice (13). At present, the gold standard for the diagnosis of ovarian cancer is surgical pathological examination, but its hysteresis of the method makes ovarian cancer difficult to be diagnosed in the early stage, thereby delaying treatment.CA125 and CA199 are commonly used clinical tumor markers, and ultrasound and $\mathrm{CT}$ are also commonly used clinical diagnostic methods (14,15). The combined detection of CA125, CA199, ultrasound and CT is costly with complicated procedures. Therefore, in this investigation, CA125 combined with CA199 was compared with ultrasound combined with $\mathrm{CT}$, in order to find a more useful reference for the early diagnosis of ovarian cancer.

In the present study, levels of serum CA125 and CA199 in 168 ovarian cancer patients were first investigated. Results showed that patients in malignant and benign group had significantly higher serum CA125 and CA199 levels than those in normal group, with statistically significant differences $(\mathrm{P}<0.05)$, and patients in malignant group had significantly higher serum CA125 and CA199 levels than those in benign group $(\mathrm{P}<0.05)$. This suggests that CA125 and CA199 have certain diagnostic values for ovarian cancer, consistent with the findings of Guo et al (16). Diagnostic values of CA125 and CA199 alone and in combination, and ultrasound and CT alone and in combination in ovarian cancer were further analyzed. Studies have proved that the accuracy of serum CA125 and CA199 alone in diagnosis of ovarian cancer is lower than that of CA125 combined with CA199 $(17,18)$, and accuracy of ultrasound and CT alone was lower than that of ultrasound combined with CT (19). This is in line with our experimental conclusion that the accuracy of CA125 and CA199 alone in diagnosis was low. It may be due to the fact that CA125 is increased in other gynecological diseases, and CA199 has different sensitivity to different pathological types, thereby affecting the accuracy of diagnosis (20). The advantage of ultrasound examination is that the color Doppler blood flow readings in ultrasound causes the high-speed and low-impedance blood flow in lumps more easily to be detected. Its limitation is that solid lumps with a diameter below $1 \mathrm{~cm}$ are not easy to be detected. Therefore, ultrasound examination has certain limitations in the detection of early ovarian cancer (21). The advantages of CT lie in its high spatial resolution and density resolution, which locate specific diagnostic sites. However, ovarian cancer may also be expressed as cystic lumps. Its solid component is not obvious and it is easily misdiagnosed as a cystic tumor, so the use of CT is limited $(22,23)$. In the present study, sensitivity, specificity and diagnostic coincidence rate of ultrasound combined with $\mathrm{CT}$ in the diagnosis of ovarian cancer were higher than those of ultrasound and CT alone. Comparison of diagnostic values between CA125 combined with CA199 and ultrasound combined CT in ovarian cancer was further investigated. Results showed that the sensitivity, specificity and diagnostic coincidence rate of CA125 combined with CA199 in the diagnosis of ovarian cancer were lower than those of ultrasound combined with CT. Misdiagnosis rate and missed diagnosis rate of CA125 combined with CA199 were higher than those of ultrasound combined with $\mathrm{CT}$. This indicates that the diagnostic value of ultrasound combined with $\mathrm{CT}$ is higher than that of AC125 combined with CA199 in ovarian cancer. In staging diagnosis, positive rate of ultrasound combined with CT in early diagnosis of ovarian cancer was higher than that of CA125 combined with CA199, indicating that in diagnosis of early ovarian cancer, ultrasound combined with CT is more significant than CA125 combined with CA199. Besides, ultrasound combined with CT is more easily accepted by general public because of its features of non-invasiveness and wide application scope, which has a better screening effect on ovarian cancer.

Although a large number of studies have respectively reported the diagnosis of ovarian cancer by CA125 combined with CA199 and ultrasound combined with CT, few studies have compared these two methods. In summary, ultrasound combined with $\mathrm{CT}$ has a higher diagnostic value than serum CA125 combined with CA199 in ovarian cancer, which can be used as a routine measure for ovarian cancer screening in clinical practice. With the continuous improvement of imaging technology, the accuracy of the diagnosis of ovarian cancer, especially the early diagnosis, will continue to increase. However, in this study, the four methods for combined diagnosis were not compared, so there were certain limitations. Moreover, a correlation analysis was not performed in this study. Therefore, these four methods combined and other diagnostic methods such as MRI need still to be compared in the diagnosis in order to provide reference for a suitable, economical and efficient diagnostic method.

\section{Acknowledgements}

Not applicable. 


\section{Funding}

No funding was received.

\section{Availability of data and materials}

The datasets used and/or analyzed during the present study are available from the corresponding author on reasonable request.

\section{Authors' contributions}

BG wrote the manuscript. BG and WL collected and analyzed the general data of patients. SL and YC recorded and analyzed CA125 and CA199 levels. JL interpreted ultrasound combined and CT result. All the authors read and approved the final manuscript.

\section{Ethics approval and consent to participate}

The study was approved by the Ethics Committee of Jining No. 1 People's Hospital (Jining, China). Patients who participated in this research had complete clinical data. The signed informed consents were obtained from the patients or the guardians.

\section{Patient consent for publication}

Not applicable.

\section{Competing interests}

All the authors declare that they have no competing interests.

\section{References}

1. Coburn SB, Bray F, Sherman ME and Trabert B: International patterns and trends in ovarian cancer incidence, overall and by histologic subtype. Int J Cancer 140: 2451-2460, 2017.

2. Barnett R: Ovarian cancer. Lancet 387: 1265, 2016.

3. Hellstrom I and Hellstrom KE: SMRP and HE4 as biomarkers for ovarian carcinoma when used alone and in combination with CA125 and/or each other. Adv Exp Med Biol 622: 15-21, 2008 .

4. Huang XM, Zhao YM, Pu Z-Y and Yu HY: Clinical value of combined detection of serum CA125, HE4 and MMP-9 in early diagnosis of ovarian cancer. Labeled Immunoassays Clin Med 9 : 1029-1032, 2016 (In Chinese).

5. Zhang Y, Jiang L and Song L: Meta-analysis of diagnostic value of serum carbohydrate antigen 199 in pancreatic cancer. Minerva Med 107: 62-69, 2016

6. Zheng LE, Qu JY and He F: The diagnosis and pathological value of combined detection of HE4 and CA125 for patients with ovarian cancer. Open Med (Wars) 11: 125-132, 2016.

7. Ietimalar H, Koksal A, Chelik N, Kasap B, Chukurova K and Iuldarum A: Evaluation of mammography and risk of developing breast cancer in patients with ovarian or endometrial cancer. Akush Ginekol (Sofiia) 48: 23-30, 2009 (In Bulgarian).
8. Castellucci P, Perrone AM, Picchio M, Ghi T, Farsad M, Nanni C, Messa C, Meriggiola MC, Pelusi G, Al-Nahhas A, et al: Diagnostic accuracy of ${ }^{18} \mathrm{~F}-\mathrm{FDG} \mathrm{PET} / \mathrm{CT}$ in characterizing ovarian lesions and staging ovarian cancer: correlation with transvaginal ultrasonography, computed tomography, and histology. Nucl Med Commun 28: 589-595, 2007.

9. Schirpenbach C, Seiler L, Maser-Gluth C, Beuschlein F, Reincke $\mathrm{M}$ and Bidlingmaier $\mathrm{M}$ : Automated chemiluminescence-immunoassay for aldosterone during dynamic testing: Comparison to radioimmunoassays with and without extraction steps. Clin Chem 52: 1749-1755, 2006.

10. Wilailak S, Chan KK, Chen CA, Nam JH, Ochiai K, Aw TC, Sabaratnam S, Hebbar S, Sickan J, Schodin BA, et al: Distinguishing benign from malignant pelvic mass utilizing an algorithm with HE4, menopausal status, and ultrasound findings. J Gynecol Oncol 26: 46-53, 2015.

11. Kim CK, Park BK, Choi JY, Kim BG and Han H: Detection of recurrent ovarian cancer at MRI: comparison with integrated PET/CT. J Comput Assist Tomogr 31: 868-875, 2007.

12. Ali AT: Fertility drugs and ovarian cancer. Curr Cancer Drug Targets 18: 567-576, 2018

13. Moore RG, Jabre-Raughley M, Brown AK, Robison KM, Miller MC, Allard WJ, Kurman RJ, Bast RC and Skates SJ: Comparison of a novel multiple marker assay vs the Risk of Malignancy Index for the prediction of epithelial ovarian cancer in patients with a pelvic mass. Am J Obstet Gynecol 203: e221-226, 2010

14. Shen Y and Li L: Serum HE4 superior to CA125 in predicting poorer surgical outcome of epithelial ovarian cancer. Tumour Biol 37: 14765-14772, 2016.

15. Fischerova D and Burgetova A: Imaging techniques for the evaluation of ovarian cancer. Best Pract Res Clin Obstet Gynaecol 28: 697-720, 2014.

16. Guo J, Yu J, Song X and Mi H: Serum CA125, CA199 and CEA combined detection for epithelial ovarian cancer diagnosis: A meta-analysis. Open Med (Wars) 12: 131-137, 2017.

17. Duffy MJ, Bonfrer JM, Kulpa J, Rustin GJ, Soletormos G, Torre GC, Tuxen MK and Zwirner M: CA125 in ovarian cancer: European Group on Tumor Markers guidelines for clinical use. Int J Gynecol Cancer 15: 679-691, 2005.

18. Zhang Q, Wang CR, Yu JP, Ma Q and Xu WW: The Establishment of an HE4-CLIA Method and the Combined Analysis of HE4 and CA125 in Ovarian Cancer. J Clin Lab Anal 30: 709-718, 2016.

19. Iyer VR and Lee SI: MRI, CT, and PET/CT for ovarian cancer detection and adnexal lesion characterization. AJR Am J Roentgenol 194: 311-321, 2010.

20. Wang K, Wang L, Cheng S J and Leng W: Diagnostic value of combined detection of serum CA125 and CA199 for ovarian cancer. Chinese J Lab Diagnosis 4: 574-576, 2014 (In Chinese).

21. Xu X-M and Jiang XS: Ultrasound combined with CA125 and CEA in the diagnosis of ovarian cancer. Chinese J Clin Oncol Rehab 9: 1059-1061, 2016 (In Chinese).

22. Rao A and Carter J: Ultrasound and ovarian cancer screening: is there a future? J Minim Invasive Gynecol 18: 24-30, 2011.

23. Schmidt S, Meuli RA, Achtari C and Prior JO: Peritoneal carcinomatosis in primary ovarian cancer staging: comparison between MDCT, MRI, and 18F-FDG PET/CT. Clin Nucl Med 40: 371-377, 2015.

This work is licensed under a Creative Commons Attribution-NonCommercial-NoDerivatives 4.0 International (CC BY-NC-ND 4.0) License. 